\title{
Production and Quality Characteristics of a Probiotic Beverage from Watermelon (Citrullus lanatus)
}

\author{
Katlego M. Masebe ${ }^{1}$, and Oluwafemi A. Adebo ${ }^{1 *}$
}

\begin{abstract}
Watermelon is a tropical fruit indigenous to Africa and prone to quick deterioration due to prevailing environmental conditions in Africa. Processing such fruit can mitigate post-harvest losses, increase its shelf life as well as to add variety in products made from it. Functional foods (particularly probiotics) are gaining more recognition due to their potential positive effects on human health and are promoted as gut-friendly products. A probiotic beverage was thus produced using a probiotic lactic acid bacteria strain. Fresh watermelon was cut and juice extracted was then aliquoted into two parts of which one was pasteurized. Both aliquots were differently fermented and analyzed for quality indices. Using the $72 \mathrm{~h}$ fermented aliquot, a beverage was developed and subsequently analyzed, this had a $\mathrm{pH}$, viscosity, total dissolvable solid and titratable acidity of 3.68 , $0.314 \mathrm{~Pa} / \mathrm{s}, 7.6^{\circ} \mathrm{Brix}$ and $0.66 \mathrm{~g} / \mathrm{L}$, respectively. This novel beverage suggests an alternative source of probiotics to intending consumers and could help reduce wastage associated with this produce.
\end{abstract}

Keywords - beverage, lactic acid bacteria, probiotics, watermelon

\section{INTRODUCTION}

Watermelon (Citrullus lanatus) is a fairly utilized fruit [1]. It is a member of the Cucurbitaceous family, thus making it closely related to vegetables such as pumpkin, cucumber, squash and cantaloupe [2]-[5]. Watermelon is extremely juicy as about $90 \%$ of its content is water [3], [6]. It has a variety of colours ranging from orange, yellow, red/pink and white. The name watermelon is associated with the deep red/pink coloured fruit [3]. The fruit is consumed worldwide, with China being the world largest producer with approximately 79 million tonnes [7]. Besides consuming watermelon as a fresh fruit, there are some food products that contain the fruit or its extract. The fruit flesh can be added to a fruit salad or its extract can be found in products such as flavoured gummy candy, flavoured soft drinks, wine, flavoured tea, flavoured chewing gums, fruit concentrate, juice and pickled watermelon rind [8].

Fermentation is a process where carbohydrates are converted to alcohol, carbon dioxide and organic acids carried out by microorganisms. Fermentation results in preservation, where the product lasts longer than the initial substrate, i.e. its natural

K. Masebe is with the Department of Biotechnology and Food Technology, Faculty of Science, University of Johannesburg, P.O. Box 17011, Doornfontein Campus, Gauteng, South Africa.

O. A. Adebo is with the Department of Biotechnology and Food Technology, Faculty of Science, University of Johannesburg, P.O. Box 17011, Doornfontein Campus, Gauteng, South Africa. state (e.g. grape fruit and wine). Depending on the mode of the process, fermentation can either be natural (spontaneous) or induced (controlled) [9], [10]. Natural (spontaneous) fermentation can both be desirable (when it is intended) and undesirable (spoilage; when not intended). In spontaneous fermentation, it is difficult to understand the capability and requirement of each species involved in the process. The disadvantages associated with natural/spontaneous fermentation have led to the need for a better, safer and efficient way for the fermentation process. This has thus led to the development of specific strains and starter cultures for the fermentation of foods to obtain desired products [11]-[13]. Sequel to their prevalence and effectiveness in fermented foods, lactic acid bacteria (LABs) are the most significant strains used for controlled fermentation. While other LABs are known, $L$. fermentum have been specifically shown to possess probiotic characteristics and have suitable fermenting properties [14]-[17], and can therefore be used to produce probiotic drinks.

According to [18] concerted efforts are being geared towards the search for probiotics as an effective strategy for health promotion and disease prevention. As defined by Food and Agriculture Organization (FAO), a probiotic is "a live microorganism which confers a health benefit to the host, when administered in adequate amounts" [19]. While there are various available probiotics in the market, this study explored the possibility of developing a probiotic product from watermelon and investigating its properties thereof.

\section{MATERIALS AND METHODS}

Ripe watermelons (Citrullus lanatus) were purchased at the City Deep Fresh Produce Market in Johannesburg, Gauteng, South Africa. Upon arrival the fruit was washed with distilled water and sanitized with $70 \%$ ethanol to reduce chances of cross contamination from wash water to the mesocarp during cutting. Washed watermelon was cut and the exocarp, mesocarp and seeds were separated. Seeds and exocarp were discarded while the mesocarp was juiced using juice extractor (DEFY Model JE210S, Japan). The mesh was discarded while the pink/red juice was further used in this study. The aliquot was then divided into two parts, one was pasteurized and the other was left raw. The pasteurization was carried out for $30 \mathrm{~min}$ at $63^{\circ} \mathrm{C}$. Both raw and pasteurized juices were further divided into two 
parts, one was naturally fermented while the other was fermented using a probiotic strain (Lactobacillus fermentum) obtained from Anatech Instruments (Pty) Ltd, Johannesburg, South Africa. The extracted juice were inoculated with a probiotic L. fermentum starter culture and incubated at $37^{\circ} \mathrm{C}$ for 24 (day 1), 48 (day 2), 72 (day 3), 96 (day 4) and $120 \mathrm{hr}$ (day 5). Natural fermentation was done in a similar way, but without the L. fermentum strain.

\section{A. Analytical Tests}

\section{Titratable Acidity (TTA)}

Using the method described by [20], the TTA of all liquids (raw, naturally fermented and LAB fermented) was analyzed by pouring $25 \mathrm{~mL}$ of the juice into $250 \mathrm{~mL}$ volumetric flask and filling to the mark with distilled water. The solution was vigorously shaken and transferred into a $100 \mathrm{~mL}$ beaker, which was then titrated with $0.119 \mathrm{M} \mathrm{NaOH}$ until it reaches the $\mathrm{pH}$ of 8.1. The results were expressed as lactic acid concentration $(\mathrm{g} / \mathrm{L})$, the process was performed in triplicate.

2. Total Dissolve Solid (TDS)

The Hanna digital refractometer for brix analysis in foods (HI96801, South Africa) was used to analyze the soluble solid content of the raw juice, fermented juice and the final product. The juice was well shaken and a drop of this was added to the stage of the refractometer and result obtained was expressed as ${ }^{\circ}$ Brix.

3. $\mathrm{pH}$ Measurement

Measurements of $\mathrm{pH}$ were conducted using a $\mathrm{pH}$ meter from Hanna Instrument HI8424 portable pHmeter, South Africa. The $\mathrm{pH}$ of raw, naturally fermented, LAB fermented aliquots and final product were all measured in triplicate.

4. Fourier Transmission-Infrared (FTIR) Spectroscopy

Using the method of [21], infrared spectra of the freeze-dried juice samples were obtained using a FTIR spectrophotometer (Thermo Fisher Scientific Inc. USA]. Before the samples was mounted on the instrument, background spectra were collected before samples and recorded with characteristic peaks in wave numbers from 450 to $4000 \mathrm{~cm}^{-1}$ at 32 runs per scan.

\section{Microbiological Analysis}

Into a sterile test-tube containing $9 \mathrm{ml}$ sterile distilled water, 1 $\mathrm{mL}$ of each sample (natural and controlled fermented aliquots) was added and mixed using a vortex (Model K-550-GE, Scientific Industries, Inc, Bohemia, NY, USA). Tenfold serial dilutions were performed on the fermented aliquots [22] and inoculated onto Nutrient agar and MRS agar plates. These were respectively incubated for 24 and $48 \mathrm{~h}$ at $37{ }^{\circ} \mathrm{C}$ [20]. After incubation, colonies were counted and colony forming units were estimated by: $\mathrm{CFU} / \mathrm{mL}=($ Number of colonies $\times$ Dilution factor)/(Volume of culture plated)

6. Protein, Fat, Dietary Fibre and Carbohydrate

The [23] method was used for the determination of protein, fat, dierary fibre and carbohydrate.

\section{Total Sodium}

For total sodium, the automatic potentiometric titrator was used. $10 \mathrm{~mL}$ of sample was measured into a $200 \mathrm{~mL}$ flask and 90 $\mathrm{mL}$ of distilled water was added and a stirred with magnetic stirrer. The sample was titrated with $0.1 \mathrm{~mol} / \mathrm{LAgNO}_{3}$ [24]. This was done in triplicate.

8. Viscosity Determination
Using the Thermo Scientific HAAKE Falling Ball Viscometer (Model: 8000009, EW-08708-51 USA), a sample of $200 \mathrm{~mL}$ was cooled to $26^{\circ} \mathrm{C}$ and poured into a viscometer cylinder, then a ball was released, and the time taken for the ball to reach the lower part of the cylinder using gravitational force was recorded and viscosity recorded.

\section{B. Sensory Evaluation of Formulated Beverage}

A beverage was formulated from the fermented juice and this was used to determine if the product was accepted by prospective consumers. Consumer acceptability test was done by using a total of 50 panelists comprising of staff and students of the Department of Biotechnology and Food Technology, University of Johannesburg, South Africa. A hedonic scale was used to assess the acceptability of the product based on color, sweetness, flavor, taste, overall likeness, and satisfaction to consumers. Products was rated from 1-5, with 1 being extremely dislike and 5 being extremely like.

\section{Statistical Analysis}

All other experiments were performed in triplicates and subsequent data generated were expressed as average \pm standard deviation. Duncan test was used to determine significant differences among the means. Principal Component Analysis (PCA) and Cluster Analysis (CA) on the FTIR data were done on Unscrambler X statistical software version 10.4.2 (Camo software, Oslo, Norway) [25].

\section{RESULT AND DISCUSSION}

\section{A. $p H$ and Titratable Acidity (TTA)}

Table I indicates the average value of the changes that occurring in $\mathrm{pH}$ on both raw, natural and LAB fermented juice. The $\mathrm{pH}$ of naturally fermented juice has decreased from 6.19 to 3.73 on day 3 but started increasing from day 4 to $\mathrm{pH} 5$ on day 5 . This is an indication of the end of fermentation that fermentable sugars are depleted, and fermenting microorganism are dying off [26]. On a contrary, both the pasteurized and unpasteurized juice fermented with L. fermentum showed a steady decrease in the $\mathrm{pH}$, from 6.19 to 3.65 on day 5 , thus indicating that fermentation was still taking place till the last day of fermentation. While continuous reduction of the $\mathrm{pH}$ values in the L. fermentum samples could suggest the viability of these strains during the fermentation process, decreased at longer fermentation times, can be attributed to the fact that the fermenting microorganisms in the naturally fermented samples have entered their decline phase, causing a reduction in microbial activity and subsequent compounds liberated. Such observation with naturally fermented samples have also been ascribed to initially low numbers of LABs causing longer lag periods, consequently resulting in reduced metabolic and microbial activity [27]. 
Changes OCCuRring In PH, TdS AND Total Plate Count (TPC) ON RAW, NATURAL AND LAB FERMENTED JUICE

\begin{tabular}{|c|c|c|}
\hline Samples & $\mathrm{pH}$ & TDS $\left({ }^{\circ}\right.$ Brix $)$ \\
\hline \multicolumn{3}{|l|}{$\mathrm{Laf}^{\phi}$} \\
\hline Day 1 & $5.10^{\mathrm{k}} \pm 0.00$ & $8.53^{j} \pm 0.06$ \\
\hline Day 2 & $3.71^{\mathrm{d}} \pm 0.00$ & $8.03^{\mathrm{i}} \pm 0.06$ \\
\hline Day 3 & $3.69^{\mathrm{d}} \pm 0.00$ & $7.77^{\mathrm{h}} \pm 0.06$ \\
\hline Day 4 & $3.63^{\mathrm{b}} \pm 0.00$ & $4.00^{\mathrm{b}} \pm 0.00$ \\
\hline Day 5 & $3.48^{\mathrm{a}} \pm 0.00$ & $3.60^{\mathrm{a}} \pm 0.00$ \\
\hline \multicolumn{3}{|l|}{$\mathrm{LafP}^{\alpha}$} \\
\hline Day 1 & $4.19^{\mathrm{g}} \pm 0.01$ & $10.50^{\mathrm{q}} \pm 0.00$ \\
\hline Day 2 & $3.75^{\mathrm{e}} \pm 0.00$ & $10.20^{\mathrm{p}_{ \pm}} .00$ \\
\hline Day 3 & $3.70^{\mathrm{d}} \pm 0.01$ & $8.73^{\mathrm{k}} \pm 0.12$ \\
\hline Day 4 & $3.69^{\mathrm{d}} \pm 0.01$ & $8.47^{\mathrm{j}} \pm 0.06$ \\
\hline Day 5 & $3.64^{\mathrm{c}} \pm 0.01$ & $5.63^{\mathrm{c}} \pm 0.06$ \\
\hline \multicolumn{3}{|l|}{$\mathrm{NF}^{\beta}$} \\
\hline Day 1 & $5.14^{1} \pm 0.01$ & $7.80^{\mathrm{h}} \pm 0.00$ \\
\hline Day 2 & $3.75^{\mathrm{e}} \pm 0.01$ & $7.67^{\mathrm{g}} \pm 0.06$ \\
\hline Day 3 & $3.68^{\mathrm{d}} \pm 0.01$ & $7.57^{\mathrm{f}} \pm 0.06$ \\
\hline Day 4 & $4.96^{\mathrm{i}} \pm 0.00$ & $7.20^{\mathrm{e}} \pm 0.00$ \\
\hline Day 5 & $5.11^{\mathrm{k}} \pm 0.00$ & $7.03^{\mathrm{d}} \pm 0.06$ \\
\hline \multicolumn{3}{|l|}{$\mathrm{NFP}^{\S}$} \\
\hline Day 1 & $5.61^{\mathrm{m}_{ \pm 0}} \pm 0.01$ & $9.93^{\circ} \pm 0.06$ \\
\hline Day 2 & $3.89^{\mathrm{f}} \pm 0.00$ & $9.37^{\mathrm{n}} \pm 0.06$ \\
\hline Day 3 & $3.73^{\mathrm{e}} \pm 0.01$ & $9.13^{\mathrm{m}_{ \pm}} \pm .12$ \\
\hline Day 4 & $4.32^{\mathrm{h}} \pm 0.02$ & $8.90^{1} \pm 0.00$ \\
\hline Day 5 & $5.00^{\mathrm{j}_{ \pm 0} .01}$ & $8.10^{\mathrm{i}} \pm 0.06$ \\
\hline Raw & $6.19^{\mathrm{n}} \pm 0.06$ & $8.67^{\mathrm{k}} \pm 0.06$ \\
\hline Pasteurized & $6.20^{\circ} \pm 0.00$ & $10.13^{\mathrm{p}_{ \pm}}+12$ \\
\hline
\end{tabular}

Each value is a mean \pm standard deviation of triplicates. Means with no common letters within a column significantly differ $(\mathrm{p}<0.05) .{ }^{\square} \mathrm{Laf}-$ lactic acid fermented; ${ }^{\alpha} \mathrm{LafP}$-pasteurized lactic fermented; ${ }^{\beta} \mathrm{NF}$-natural fermented; ${ }^{\S} \mathrm{NFP}-$ pasteurized natural fermented.

As observed in Fig. 1, TTA of the L. fermentum juices kept increasing from 0.36 (on day 0 ) to $0.95 \mathrm{~g} / \mathrm{L}$ (on day 5) and a similar trend was also observed for the naturally fermented juice, but with a lower TTA value (least was $0.26 \mathrm{~g} / \mathrm{L}$ on day 5). There was a relationship between the $\mathrm{pH}$ values earlier obtained and the TTA values, as increase in acidity was observed to be inversely proportional to the decrease in $\mathrm{pH}$. This could thus suggest that as fermentation of watermelon with or without the probiotic $L$. fermentum strain resulted in the production of organic acids. The increase of acidity in lactic acid fermented aliquots suggest that there are more organic acids formed in the controlled fermentation than in natural fermentation, a similar trend observed for the $\mathrm{pH}$ values. This is because the $L$. fermentum strains are hetero fermentors, this suggest that they produce other organic material beside lactic acids, such as acetic acids, ethyl alcohol and carbon dioxide which also affect the value of acidity in the aliquots.

Fig. 1. Titratable acidity for fermented juice in different days Laf - lactic acid fermented; LafP - pasteurized lactic fermented; NF -natural fermented; NFP - pasteurized natural fermented.

\section{TTA VS DAYS OF FERMENTATION}

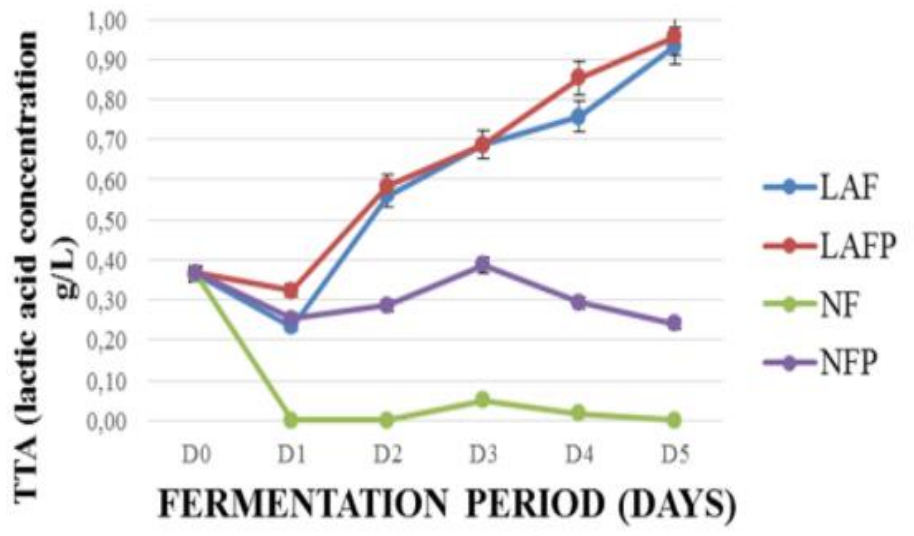

Fig. 1. Titratable acidity for fermented juice in different days Laf - lactic acid fermented; LafP - pasteurized lactic fermented; NF -natural fermented; NFP -pasteurized natural fermented.

\section{B. Total Dissolve Solids}

According to [28], decrease in TDS indicates the utilization of fermentable sugars by the microorganism as carbon and energy sources, to subsequently produce metabolites. Significantly $(p \leq 0.05)$ lower TDS values of the LAB-fermented samples suggest an increased utilization of available sugars in the aliquots. The least TDS of 3.60 was obtained after the $5^{\text {th }}$ day of fermentation, with a corresponding significantly $(\mathrm{p} \leq 0.05)$ lowest $\mathrm{pH}$ value of 3.48 . This further suggests increased microbial activities and utilization of the carbon sources by the L. fermentum strain during the production of the aliquot.Available fermentable sugars in watermelon include fructose, fructan, glucose and polyol [29], all of which could have been effectively utilized by the probiotic strain. Furthermore, it could be suggested that the presence of other microorganisms dominating the microflora of the naturally fermented samples caused relatively higher TDS values (Table I). While the use of L. fermentum would have led to better adaptability and dominance from the beginning of the fermentation producing, leading to significantly lesser residual fermentable sugars, the controlled fermentation process, competitive action of the plethora of microorganisms during the natural fermentation hindered this, resulting in higher amounts of residual sugars.

\section{C.Microbiological Study}

Microbial analysis was performed on the different aliquots to monitor the microbial growth or otherwise with fermentation days. As shown in Table II, as the day progresses the amount of $\mathrm{CFU} / \mathrm{mL}$ decrease due to the depletion of sugar, aging of microorganism and accumulation of waste in the medium. The relatively higher microbial counts obtained on the plate count agar suggests a broad diversity of bacteria in the fermented aliquots. This also indicates that natural (spontaneous) fermentation results from a competitive action of different endogenous microorganisms [30]. The higher mean counts of the viable bacteria and LAB of samples fermented with starter cultures suggest that the starter culture dominated and 
constituted major portion of the viable bacteria growing on the samples.

TABLE II

MicrobiologicAl ANALYSIS OF FERMENTEd JUICE

\begin{tabular}{|c|c|c|c|}
\hline Days & $\begin{array}{l}\text { Type of } \\
\text { fermentation }\end{array}$ & $\begin{array}{l}\text { Total plate count } \\
\mathrm{CFU} / \mathrm{mL}\left(\times 10^{10}\right)\end{array}$ & $\begin{array}{l}\text { Anaerobic plate } \\
\text { count (MRS) } \\
\text { CFU } / \mathrm{mL}\left(\times 10^{10}\right)\end{array}$ \\
\hline \multirow[t]{4}{*}{1} & $\mathrm{NF}^{\beta}$ & $5^{b} \pm 0.03$ & $3^{\mathrm{a}} \pm 0.16$ \\
\hline & $\mathrm{NFP}^{\S}$ & $3^{\mathrm{a}} \pm 0.25$ & $4^{\mathrm{ab}} \pm 0.07$ \\
\hline & $\mathrm{Laf}^{\phi}$ & $7^{\mathrm{bc}} \pm 0.11$ & $5^{\mathrm{b}} \pm 0.05$ \\
\hline & $\operatorname{LafP}^{\alpha}$ & $6^{\mathrm{b}} \pm 0.34$ & $5^{\mathrm{b}} \pm 0.00$ \\
\hline \multirow[t]{4}{*}{2} & $\mathrm{NF}^{\beta}$ & $14^{\mathrm{f}} \pm 0.04$ & $11^{\mathrm{d}} \pm 0.00$ \\
\hline & $\mathrm{NFP}^{\S}$ & $9^{\mathrm{d}} \pm 0.00$ & $7^{\mathrm{bc}} \pm 0.00$ \\
\hline & $\mathrm{Laf}^{\phi}$ & $11^{\mathrm{e}} \pm 0.01$ & $9^{c} \pm 0.01$ \\
\hline & $\mathrm{LafP}^{\alpha}$ & $9^{\mathrm{d}} \pm 0.01$ & $8^{\mathrm{c}} \pm 0.00$ \\
\hline \multirow[t]{4}{*}{3} & $\mathrm{NF}^{\beta}$ & $19^{\mathrm{g}} \pm 0.00$ & $15^{\mathrm{f}} \pm 0.01$ \\
\hline & $\mathrm{NFP}^{\S}$ & $12^{\mathrm{ef}} \pm 0.00$ & $9^{c} \pm 0.01$ \\
\hline & $\mathrm{Laf}^{\phi}$ & $24 \pm 0.09$ & $18^{\mathrm{gh}} \pm 0.25$ \\
\hline & $\operatorname{LafP}^{\alpha}$ & $20^{\mathrm{gh}} \pm 0.12$ & $15^{\mathrm{f}} \pm 0.00$ \\
\hline \multirow[t]{4}{*}{4} & $\mathrm{NF}^{\beta}$ & $28 \pm 0.21$ & $17^{\mathrm{g}} \pm 0.00$ \\
\hline & $\mathrm{NFP}^{\S}$ & $16^{\mathrm{fg}_{ \pm}} \pm 0.00$ & $13^{\mathrm{de}} \pm 0.01$ \\
\hline & $\mathrm{Laf}^{\phi}$ & $26^{\mathrm{j}} \pm 0.01$ & $24^{\mathrm{j}} \pm 0.00$ \\
\hline & $\operatorname{LafP}^{\alpha}$ & $22^{\mathrm{h}} \pm 0.05$ & $20^{\mathrm{h}} \pm 0.00$ \\
\hline \multirow[t]{4}{*}{5} & $\mathrm{NF}^{\beta}$ & $20^{\mathrm{gh}} \pm 0.05$ & $17^{\mathrm{g}} \pm 0.01$ \\
\hline & $\mathrm{NFP}^{\S}$ & $15^{\mathrm{f}} \pm 0.00$ & $12^{\mathrm{d}} \pm 0.00$ \\
\hline & $\mathrm{Laf}^{\phi}$ & $24^{\mathrm{hi}} \pm 0.00$ & $26^{\mathrm{j}} \pm 0.00$ \\
\hline & $\operatorname{LafP}^{\alpha}$ & $23^{\mathrm{h}} \pm 0.01$ & $21^{\mathrm{hi}} \pm 0.00$ \\
\hline
\end{tabular}

Each value is a mean \pm standard deviation of triplicates. ${ }^{\beta} \mathrm{NF}$-natural fermented; ${ }^{\S} \mathrm{NFP}$-pasteurized natural fermented; ${ }^{\square}$ Laf- lactic acid fermented; ${ }^{\alpha}$ LafP-pasteurized lactic fermented.

\section{D.Fourier Transmission Infrared (FTIR) Spectroscopy}

FTIR analysis was performed on the obtained juices to identify possible variations in their spectra. It was envisaged that the obtained spectra would provide a wider spectrum of chemical analysis of all present metabolites in the fermented juice. Computation and chemometric steps of data pre-treatments were followed for the accumulated spectra using PCA and CA. This assisted with the interpretation and comparison of the FTIR spectral data set with the aim of evaluating biochemical events that occurred during fermentation and to discriminate and classify the samples according to clusters and groupings.

All the obtained spectra data were in the FTIR spectroscopic ranges of 3000-600 $\mathrm{cm}^{-1}$ wave number [31]. As observed from the mean plots of the average spectra of each sample (Fig. 2 (a), the significant peaks of the product after fermentation were at $774.67 \mathrm{~cm}-1$ and $816.44 \mathrm{~cm}-1$ which lies in the $\mathrm{C}-\mathrm{Cl}$ stretch alkyl halides peak of $850-550 \mathrm{~cm}^{-1}, 865.36 \mathrm{~cm}^{-1}$ represent $1^{\circ}, 2^{\circ}$ amines $(910-665 \mathrm{~cm}-1) \mathrm{N}-\mathrm{H}$, carboxylic acids group 911.56 and $1038.68 \mathrm{~cm}^{-1}$ are aliphatic amines $\left(1250-1020 \mathrm{~cm}^{-1}\right)$, alkyl halides by 1250.64 and $1353.22 \mathrm{~cm}^{-1}$ are nitro compounds and aromatic amines, aromatics, alkanes and $1^{\circ}, 2^{\circ}$ amines, amides while the last day of fermentation indicated development of aldehyde, saturated aliphatic and alkynes terminal. During natural fermentation there are alkyl halides and aromatic, carboxylic acids, aliphatic amines, nitro compounds, aromatic, ester saturated aliphatic (which lactic acid fermentation didn't contain in day 1 of the fermentation), alkanes and carboxylic acids. The last day of natural fermentation the aliquot developed the following functional groups aromatic amide, and alkyne amine. Thus, indicating that during fermentation there are functional groups forms and others terminated depending on the type of fermentation, sugar substrate and microorganism responsible for fermentation.

Using PCA, the spectra of the fermented juice samples were transformed into principal components (PCs). The first two PCs attributed for $97 \%$ of the variation (Fig. 2 (b) and differentiated the samples. From the left to the right sample are day 4 of natural fermentation, day 3 of lactic acid fermentation, day 4 lactic acid fermentation, day 5 natural fermentation, day 5 and 2 lactic acid fermentation, day 3 natural fermentation, day 1 natural fermentation, raw (un-pasteurize), pasteurize, day 2 natural fermentation and day 1 lactic acids fermentation. To further differentiate the fermented watermelon aliquots samples from each other, a third PC were used which provided an additional $2 \%$ variation in the data obtained. Cluster analysis (CA) was further used to classify the samples into groups, as a function of their clusters. This was performed to group sample based on proximity of the characteristic, to give a descriptive, theoretical and non-inferential analysis. As shown in Fig. 2 (d), the samples were classified into two major clusters, a group with red colour and the other with blue. The red colour represent the lactic acid fermented aliquots of Day 3 and the natural fermented aliquots of day 4 this two pose similar behaviour their TDS are between 8.7-8.9 which fall under the same range, $\mathrm{pH}$ is also not different from each other 3.70 and 4.32 , which means it takes 3 days for the lactic acid bacteria to lower the $\mathrm{pH}$ and use up the same nutrients as the mixture of organism in the natural fermented aliquots. The blue are the rest of the days and type of fermentation from unpasteurized, pasteurized, day 1 till day 5 of both fermentations. Even though the range of investigated parameters in the days is wide they were clustered together because they contain similar functional groups and bands. The red cluster falls under the same section in the PCA graph (left hand side) while the rest are on the far-right hand side.

\section{E. Nutritional, Physiochemical Information and Sensory Evaluation}

After subsequent analysis on the fermented juice, a product was developed from the pasteurized juice fermented with the LAB strain for 5 days. This was particularly selected due to its reduced $\mathrm{pH}$ and relatively high TTA values. Pasteurization is also particularly important and an important component in food processing. The resulting product was also evaluated for nutritional properties. 


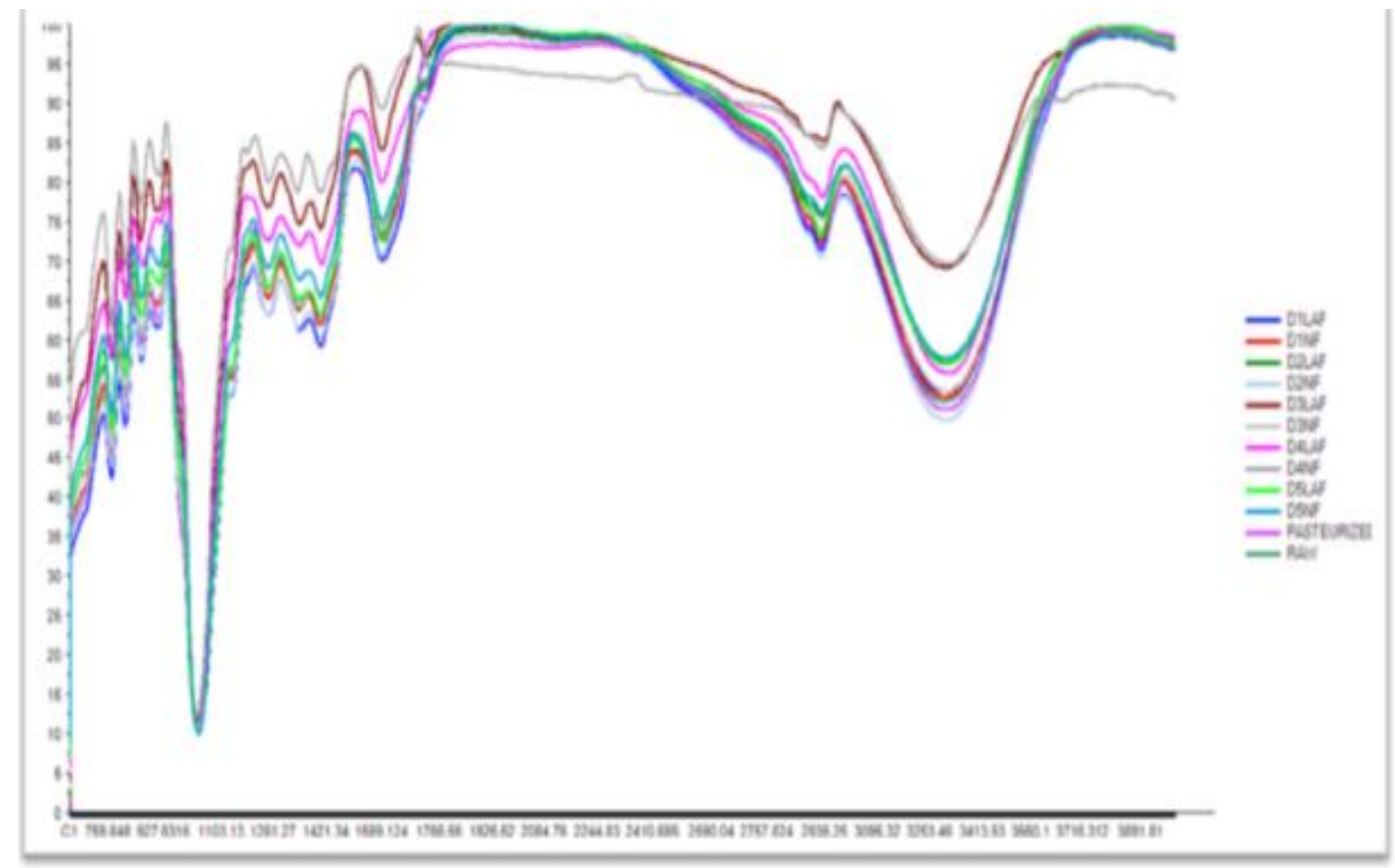

Fig. 2 (a) Average FTIR plot of fermented watermelon juice

D1LAF-Day 1 Lactic acid fermentation, D1NF-Day 1 Natural fermentation, D2LAF-Day 2 Lactic acid fermentation, D2NF-Day 2 Natural fermentation, D3LAF-Day 3 Lactic acid fermentation, D3NF-Day 3 Natural fermentation, D4LAF-Day 4 Lactic acid fermentation, D4NF-Day 4 Natural fermentation, D5LAF-Day 5 Lactic acid fermentation, D5NF-Day 5 Natural fermentation, RAW-Raw Aliquot, PASTEURIZED-Pasteurized aliquot.

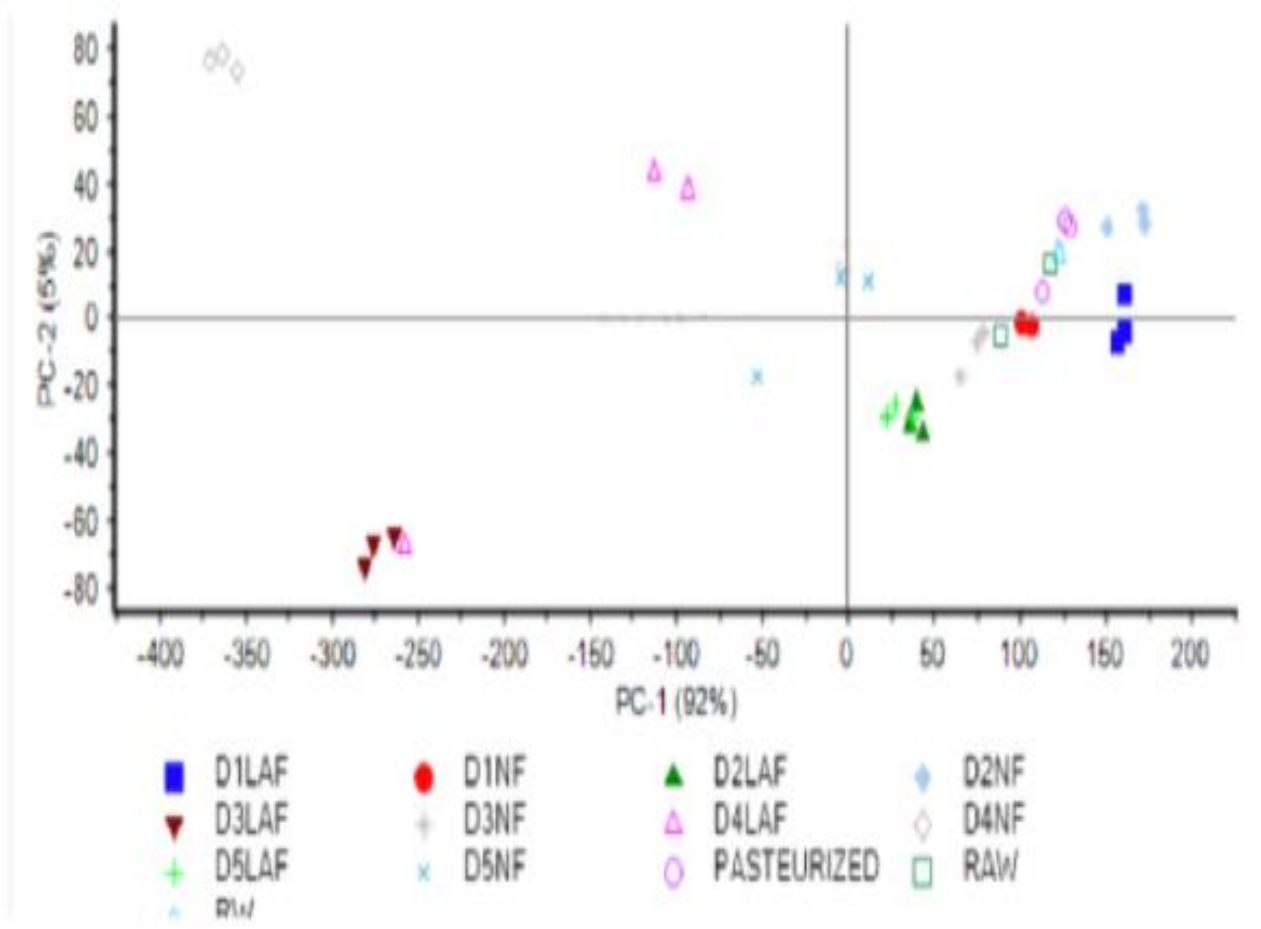

Fig. 2 (b) PCA Score plot of the fermented watermelon juice showing PC1 and PC2

D1LAF-Day 1 Lactic acid fermentation, D1NF-Day 1 Natural fermentation, D2LAF-Day 2 Lactic acid fermentation, D2NF-Day 2 Natural fermentation, D3LAF-Day 3 Lactic acid fermentation, D3NF-Day 3 Natural fermentation, D4LAF-Day 4 Lactic acid fermentation, D4NF-Day 4 Natural fermentation, D5LAF-Day 5 Lactic acid fermentation, D5NF-Day 5 Natural fermentation, RAW-Raw Aliquot, PASTEURIZED-Pasteurized aliquot. 


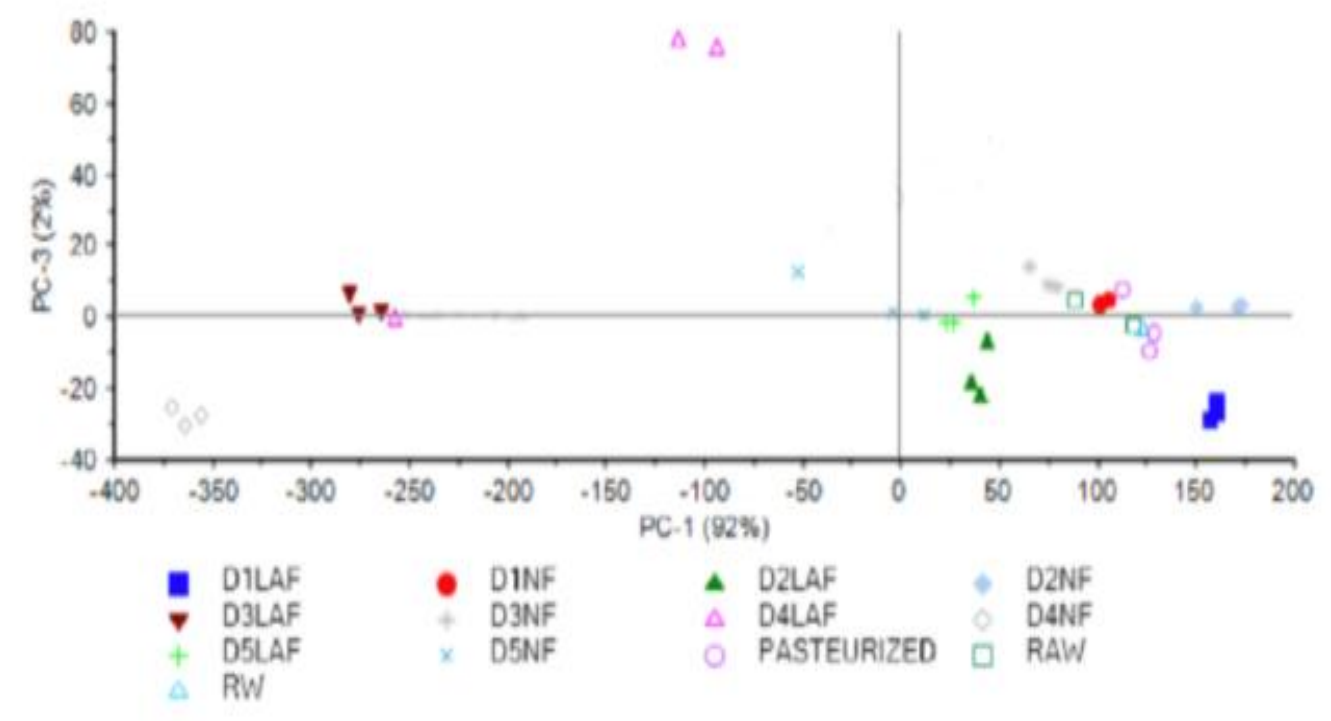

Fig. 2 (c).PCA Score plot of the fermented watermelon juice showing PC1 and PC3

D1LAF-Day 1 Lactic acid fermentation, D1NF-Day 1 Natural fermentation, D2LAF-Day 2 Lactic acid fermentation, D2NF-Day 2 Natural fermentation, D3LAF-Day 3 Lactic acid fermentation, D3NF-Day 3 Natural fermentation, D4LAF-Day 4 Lactic acid fermentation, D4NF-Day 4 Natural fermentation, D5LAF-Day 5 Lactic acid fermentation, D5NF-Day 5 Natural fermentation, RAW-Raw Aliquot, PASTEURIZED-Pasteurized aliquot.

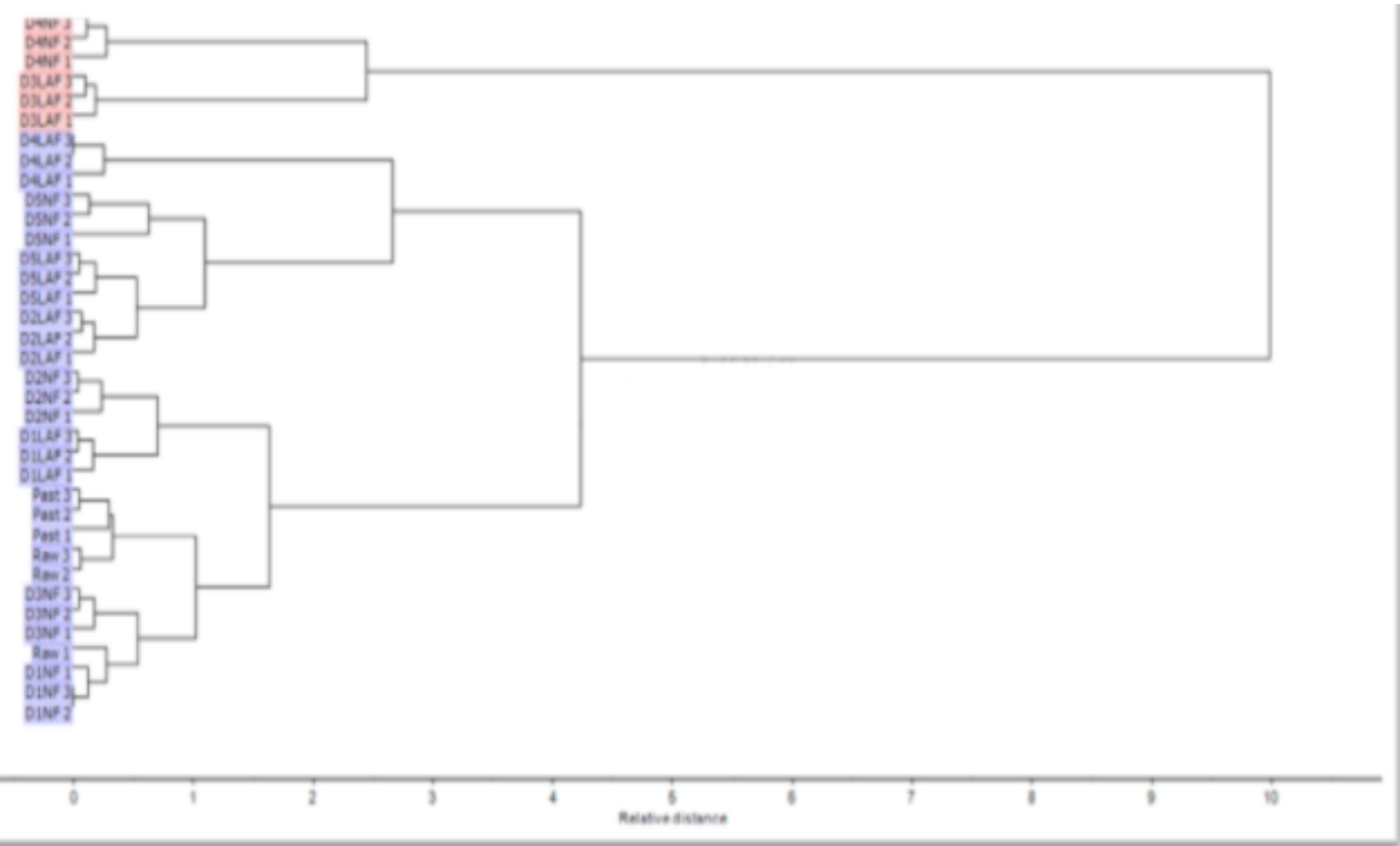

Fig. 2 (d) Cluster analysis of the fermented watermelon juice

D1LAF-Day 1 Lactic acid fermentation, D1NF-Day 1 Natural fermentation, D2LAF-Day 2 Lactic acid fermentation, D2NF-Day 2 Natural fermentation, D3LAF-Day 3 Lactic acid fermentation, D3NF-Day 3 Natural fermentation, D4LAF-Day 4 Lactic acid fermentation, D4NF-Day 4 Natural fermentation, D5LAF-Day 5 Lactic acid fermentation, D5NF-Day 5 Natural fermentation. As observed in Table III, the product contributes an energy $\mathrm{g}$ per $100 \mathrm{~mL}$ serving. Other physicochemical properties of the value of $488 \mathrm{~kJ}$ per serving and a glycaemic carbohydrate of 14 drink are presented in Table IV. Results from the sensory 
evaluation revealed that the panellists generally accepted the beverage (Table V). Although the score was less than 5 (the maximum), the least score was for the taste and overall acceptability of 3.84 each. This could be due to the "newness" of this product, as watermelon is not a common probiotic drink or juice available in the market. Although the product was not subjected to comparison test (in comparison with other juices/probiotic products), the developed probiotic beverage still has a competitive advantage.

TABLE III

NUTRITIONAL INFORMATION OF THE PROBIOTIC WATERMELON BEVERAGE

Typical nutritional information (as packed)

Serving size $(200 \mathrm{ml})$

\begin{tabular}{lll}
\hline & & \\
\hline Serving size $(200 \mathrm{ml})$ & Per $100 \mathrm{~mL}$ & Per serving size \\
\hline Energy $(\mathrm{kJ})$ & 244 & 488 \\
Protein $(\mathrm{g})$ & 1 & 2 \\
Glycaemic carbohydrate $(\mathrm{g})$ & 14 & 28 \\
Of which total sugar $(\mathrm{g})$ & 6 & 12 \\
Total fat $(\mathrm{g})$ & 0 & 0 \\
Of which saturated fat $(\mathrm{g})$ & 0 & 0 \\
Dietary fibre $(\mathrm{g})$ & 0.4 & 0.8 \\
Total sodium $(\mathrm{mg})$ & 1 & 2 \\
\hline \hline
\end{tabular}

TABLE IV

PHYSICOCHEMICAL PROPERTIES OF THE DEVELOPED BEVERAGE

\begin{tabular}{ll}
\hline \hline Product characteristics & Value \\
\hline $\mathrm{pH}$ & 3.68 \\
Viscosity & $0.314 \mathrm{~Pa} \mathrm{~s}$ \\
Total dissolvable solid & $7.6^{\circ}$ Brix \\
Titratable acidity & 0.66 Lactic acid concentration \\
& $(\mathrm{g} / \mathrm{L})$ \\
\hline \hline
\end{tabular}

TABLE V

SEnsory Test Results For The PRobiotic Watermelon BeVerage

\begin{tabular}{ll} 
Parameters & Score \\
Color & $4.32 \pm 0.62$ \\
Sweetness & $4.06 \pm 0.65$ \\
Flavor & $3.88 \pm 0.48$ \\
Taste & $3.84 \pm 0.71$ \\
Overall acceptance & $3.84 \pm 0.55$ \\
\hline
\end{tabular}


[14] E. F. Reque, A. P. Ashok, S. G. Franco, and C. R. Socco, "Isolation, identification and physiological study of Lactobacillus fermentum LPD for use as probiotic in chickens," Brazilian Journal of Microbiology, vol. 31, pp. 303-307, 2000 https://doi.org/10.1590/S1517-83822000000400012

[15] G. E. Gardiner, C. Heinemann, A. W. Bruce, D. Beuerman, and G. Reid, "Persistence of Lactobacillus fermentum RC-14 and Lactobacillus rhamnosus GR-1 but not L. rhamnosus GG in the human vagina as demonstrated by randomly amplified polymorphic DNA," Journal of Clinical and Vaccine Immunology, vol. 9, pp. 92-96, 2002. https://doi.org/10.1128/CDLI.9.1.92-96.2002

[16] G. A. Gerbaldo, C. Barberis, L. Pascual, A. Dalcero, and L. Lucila-Barberis, "Antifungal activity of two Lactobacillus strains with potential probiotic properties," FEMS Microbiology Letters, vol. 332, pp. $27-33,2012$. https://doi.org/10.1111/j.1574-6968.2012.02570.x

[17] O. A. Adebo, E. Kayitesi, F. Tugizimana, and P. B. Njobeh, "Differential metabolic signatures in naturally and lactic acid bacteria (LAB) fermented ting (a Southern African food) with different tannin content, as revealed by gas chromatography mass spectrometry (GC-MS)-based metabolomics," Food Research International, vol. 121, pp. 326-335, 2019. https://doi.org/10.1016/j.foodres.2019.03.050

[18] S. Sampaolesi, R. R. Gamba, G. L. De Antoni, and A. M. L. Pelaez, "Potentiality of yeasts obtained as beer fermentation residue to be used as probiotics," LWT-Food Science and Technology, vol. 113, 108251, 2019.

https://doi.org/10.1016/j.1wt.2019.108251

[19] FAO/WHO. Guidelines for the evaluation of probiotics in food, 2002, London, Ontario, Canada.

[20] A. Chwastek, E. Klewicka, R. Klewicki, and M. Sójka, "Lactic acid fermentation of red beet juice supplemented with waste high bush blueberry-sucrose osmotic syrup as a method of probiotic beverage production," Journal of Food Processing and Preservation, vol. 40, pp. 780-789, 2016 https://doi.org/10.1111/jfpp.12659

[21] J. A. Adebiyi, A. O. Obadina, A. F. Mulaba-Bafubiandi, O. A. Adebo, and K. Eugenie, "Effect of fermentation and malting on the microstructure and selected physicochemical properties of pearl millet (Pennisetum glaucum) flour and biscuit," Journal of Cereal Science, vol. 70, pp. 132-139, 2016. https://doi.org/10.1016/j.jcs.2016.05.026

[22] J. J. Z. Liao, and F. Duan, "Calibrating the concentration from a serial dilution process," Journal of Chemometrics, vol. 20, pp. 294-301, 2006 https://doi.org/10.1002/cem.1021

[23] AOAC (Association of Official Analytical Chemists) (2016). Official Methods of Analysis of AOAC International, $20^{\text {th }}$ Ed. (editor, Latimer, G.W).

[24] KEM Kyoto Electronics Manufacturing (May 2016). Automatic potentiometric titrator. Available: https://www.mt.com/int/en/home/products/Laboratory_Analytics_Brows e/Product_Family_Browse_titrators_main/Compact_Titration.html.

[25] O. A. Adebo, P. B. Njobeh, A. F. Mulaba-Bafubiandi, J. A. Adebiyi, Z. S. C. Desobgo, and E. Kayitesi, "Optimization of fermentation conditions for ting production using response surface methodology," Journal of Food Processing and Preservation, vol. 42, pp. 1-10, April 2018b. https://doi.org/10.1111/jfpp.13381

[26] A. Rault, M. Bouix, and C. Béal, "Fermentation $\mathrm{pH}$ influences the physiological-state dynamics of Lactobacillus bulgaricus CFL1 during pH-Controlled Culture," Applied Environmental Microbiology, vol. 75, pp. 4374-4381, 2009. https://doi.org/10.1128/AEM.02725-08

[27] O. A. Adebo, P. B. Njobeh, and E. Kayitesi, "Fermentation by Lactobacillus fermentum strains (singly and in combination) enhances the properties of ting from two whole grain sorghum types," Journal of Cereal Science, vol. 82, pp. 49-56, May 2018c. https://doi.org/10.1016/j.jcs.2018.05.008

[28] T. Ghaffara, M. Irshada, Z. Anwara, T. Aqilb, Z. Zulifqara, A. Tariqa, M. Kamrana, N. Ehsana, and S. Mehmooda, "Recent trends in lactic acid biotechnology: a brief review on production to purification," Journal of Radiation Research and Applied Sciences, vol. 7, pp. 222-229, 2012. https://doi.org/10.1016/j.jrras.2014.03.002
[29] B. Ozcelik, and M. Yavuz, "Watermelon juice: Handbook of Functional Beverages and Human Health. F. Shahidi, and C. Alasalvar, Ed. USA: CRC Press, 2016, ch. 45, pp. 553-562. https://doi.org/10.1201/b19490-48

[30] O. A. Adebo, P. B. Njobeh, J. A. Adebiyi, and E. Kayitesi, "Co-influence of fermentation time and temperature on physicochemical properties, bioactive components and microstructure of ting (a Southern African food) from whole grain sorghum," Food Bioscience, vol. 25, pp. 118-127, August 2018d. https://doi.org/10.1016/j.fbio.2018.08.007

[31] R. M. Pop, A. D. Buzoianui, I. V. Rati, and C. Socaciu, "Untargeted metabolomics for sea buckthorn (Hippophae rhamnoides ssp. carpatica) berries and leaves: fourier transform infrared spectroscopy as a rapid approach for evaluation and discrimination. Notulae Botanicae Horti Agrobotanici Cluj-Napoca, vol. 42, pp. 545-550, 2014.

https://doi.org/10.15835/nbha4229654 